\title{
Evaluation of wave propagation effects for detection of vulnerable road users
}

\author{
A. Fackelmeier, C. Morhart, and E. M. Biebl \\ Fachgebiet Höchstfrequenztechnik, Technische Universität München, Munich, Germany
}

\begin{abstract}
The protection of vulnerable road users (VRU) is a key issue of future driver assistance systems. An important point is to determine whether a VRU is visually hidden to the driver. This additional information is essential for the driver to prevent an accident or to reduce its severity. We show that by using a transponder attached at the VRU, wavelength dependent diffraction effects can be utilized to distinguish between visible and hidden VRUs. Several diffraction measurements were carried out taking place in open space areas with simple big scattering objects and in different road traffic scenarios demonstrating a reliability of nearly $100 \%$. If future driver assistance systems for detecting VRUs are complemented with automotive electronics which enable distinction between visible and hidden VRUs, accidents can be avoided.
\end{abstract}

\section{Introduction}

The protection of vulnerable road users (VRU) is a key issue of future driver assistance systems (Rasshofer, 2007). Currently, several different systems are under investigation, based on passive sensing techniques (radar, lidar, camera) as well as on cooperative sensor systems. In a cooperative system the VRU is required to wear a transponder in order to identify themselves as a VRU as well as to send a beacon signal for localization. In contrast to passive sensing, the beacon signal can also be detected if the person wearing the transponder is visually hidden, for instance by a parked car or a roadside tree. Of course, information about this situation, i.e. whether the transponder is visually hidden or not, will be of great value for alerting the driver of the car. If the person

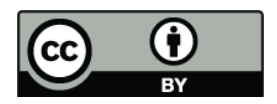

Correspondence to: A. Fackelmeier (fackelmeier@tum.de) wearing the transponder is visually hidden, the situation is much more hazardous.

Our method to identify visually hidden persons is based on dual-frequency discrimination. A transponder (according to the terms of radar technology we call it "active target") attached at the VRU emits two signals with different frequencies which are detected by the receiver in the car. If the VRU is invisible for the driver, the signals are diffracted around the scattering object and get an additional attenuation. Signals with different wavelengths experience different attenuation at the same observation angle in the shadow zone due to the wavelength dependent diffraction. The difference in signal power at the two frequencies compared to the difference at line of sight (LOS) received at the driver side is an indicator whether the emitter is hidden or not. There are many parameters to be investigated affecting the measurement result such as orientation of the VRU to the scattering object and to the receiver, height of the antennas, multipath propagation due to buildings or objects nearby and the size and shape of the scattering object which hides the VRU. Our objective was to determine whether this diffraction effect is measurable in real road traffic scenarios, how reliable a detection of hidden VRU is, and how the parameters mentioned above influence the measurement results.

\section{Wave propagation effects}

For theoretical analysis, the dimension of this scattering problem compared to the wavelength is too big for applying EM field simulation methods. The Geometrical Theory of Diffraction (GTD), first introduced by Keller in 1962 (Keller, 1962; Borovikov, 1992), is an extension to the geometrical optics which adds diffracted rays $E_{d}$ to the geometrical optics. Dimensional considerations show that the edge diffraction is the strongest besides corner and surface diffraction (Keller, 1962) and therefore, the latter are neglected. Edge

Published by Copernicus Publications on behalf of the URSI Landesausschuss in der Bundesrepublik Deutschland e.V. 


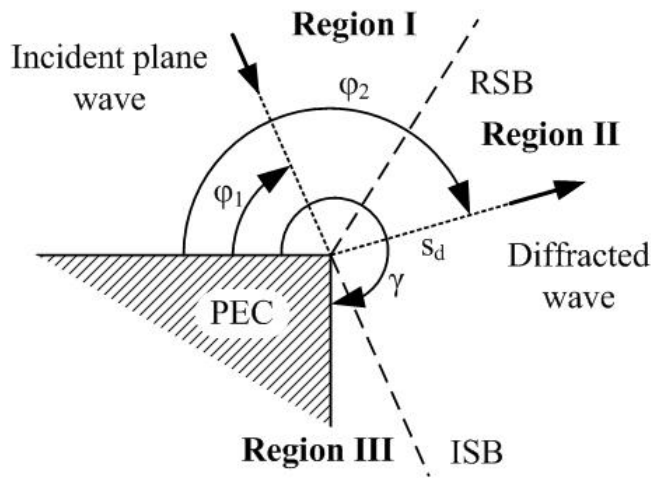

Fig. 1. Edge diffraction (ISB: Incident Shadow Boundary, RSB: Reflection Shadow Boundary).

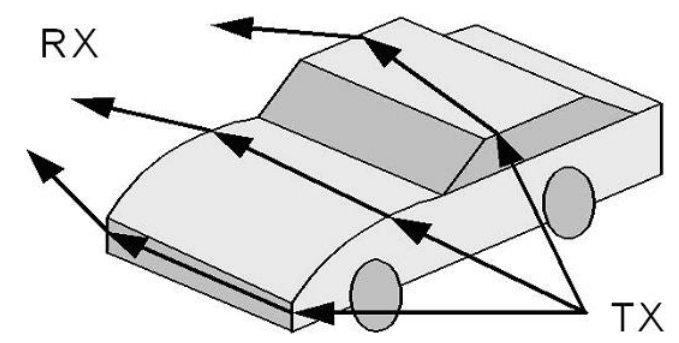

Fig. 2. Double edge diffraction at a car.

diffraction occurs with an edge radius small compared to $\lambda$. The incident wave is diffracted at the edge and spreads out as a cylindrical wave (Fig. 1).

With the diffraction coefficient $R$, the signal power difference in the shadow region can be calculated for different frequencies. $E_{i}$ is the field incident at the point of diffraction and $A\left(s_{d}\right)(=1 / \sqrt{ } s$ for edge diffraction) is the spreading factor with the distance $s_{d}$ between the scattering point and the observation point $P$ were the diffracted field strength $E_{d}$ is present.

The diffraction coefficient $R$ has to be calculated for each frequency with the angles indicated in Fig. 1. Between $f_{u}=433 \mathrm{MHz}$ and $f_{o}=2.45 \mathrm{GHz}$ the ratio in signal power of the diffracted waves is about $7.5 \mathrm{~dB}$. If there is a second edge, double edge diffraction occurs and the power difference amounts to about $15 \mathrm{~dB}$. Double diffraction occurs quite often in road traffic situations with cars as scattering objects (Fig. 2).

$$
\begin{aligned}
E_{d}(P)=R \cdot A\left(s_{d}\right) \cdot E_{i} \cdot \exp \left(-j k_{0} s_{d}\right) \\
\left.\begin{array}{l}
R_{\perp} \\
R_{\|}
\end{array}\right\}=\frac{\exp \left(-j \frac{\pi}{4}\right) \sin \left(\frac{\pi^{2}}{\gamma}\right)}{\frac{\gamma}{\pi} \sqrt{2 k_{0} \pi}} \cdot\left[\frac{1}{\cos \left(\frac{\pi^{2}}{\gamma}\right)-\cos \left(\frac{\left(\varphi_{1}-\varphi_{2}\right) \pi}{\gamma}\right)}\right. \\
\left. \pm \frac{1}{\cos \left(\frac{\pi^{2}}{\gamma}\right)+\cos \left(\frac{\left(\varphi_{1}-\varphi_{2}\right) \pi}{\gamma}\right)}\right]
\end{aligned}
$$

Multipath propagation can cause an attenuation of more than $15 \mathrm{~dB}$ which is very problematic in this application as it has

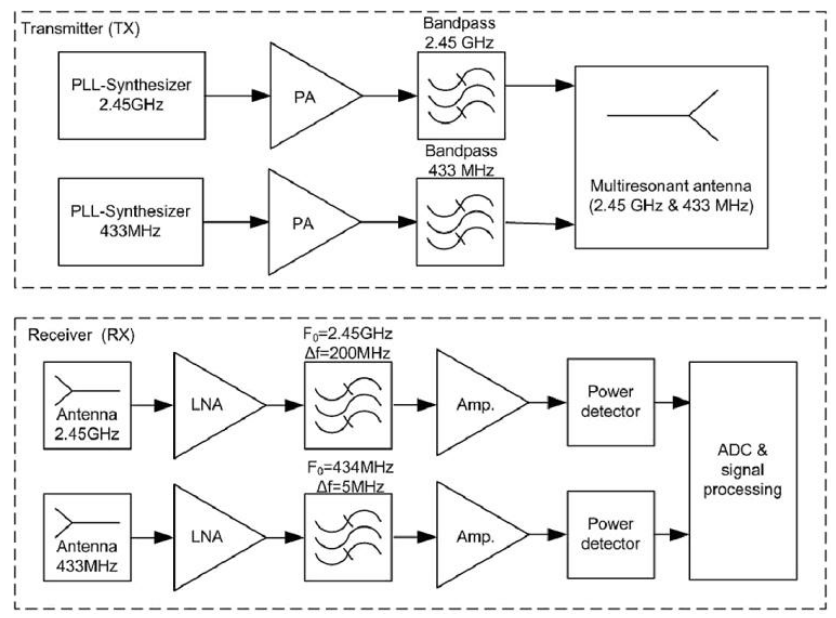

Fig. 3. Emitter (above) and receiver (below).

to be distinguished between attenuation due to scattering objects in the signal path and attenuation due to multipath propagation. In road traffic situations, multipath propagation can occur through every object near the road such as buildings, vehicles or other obstacles nearby.

\section{The RF-power measurement system}

A RF-power measuring system was developed and built up for the ISM-frequency bands at $2.45 \mathrm{GHz}$ and $433 \mathrm{MHz}$ with commercial IC devices to show that the target detection is realizable also without high-precision measurement equipment (Fig. 3). For each emitter (TX), a Phased Locked Loop (PLL) frequency stabilisation is used to avoid a frequency drift which can result in an output power variation and therefore decrease the measurement accuracy. At the receiver (RX) a high dynamic range is necessary to cover different distances of RX and TX and the additional attenuation due to the scattering objects in between. A low pass filter is implemented to flatten the interfering fast fading peaks in the signal waveform.

The transmitter antennas have to be quite omni-directional enabling detection of the target at every orientation. Other parameters like polarisation also affect the measurement results.

The small distance of the transmitting antennas to the object leads to distorted measurement results due to displacement between the antennas. This problem does not occur at the receiver which is located in a huge distance to the object. Simply adding the $2.45 \mathrm{GHz}$ signals to the $433 \mathrm{MHz}$ monopole is disadvantageous because measurements have shown that the side lobes arising at the $2.45 \mathrm{GHz}$ antenna pattern in special cases are reflected, for instance at the headlining inside the car. To overcome this problem, a multi-resonant antenna was developed which provides a nearly coinciding phase centre (Fig. 4). For the presented 


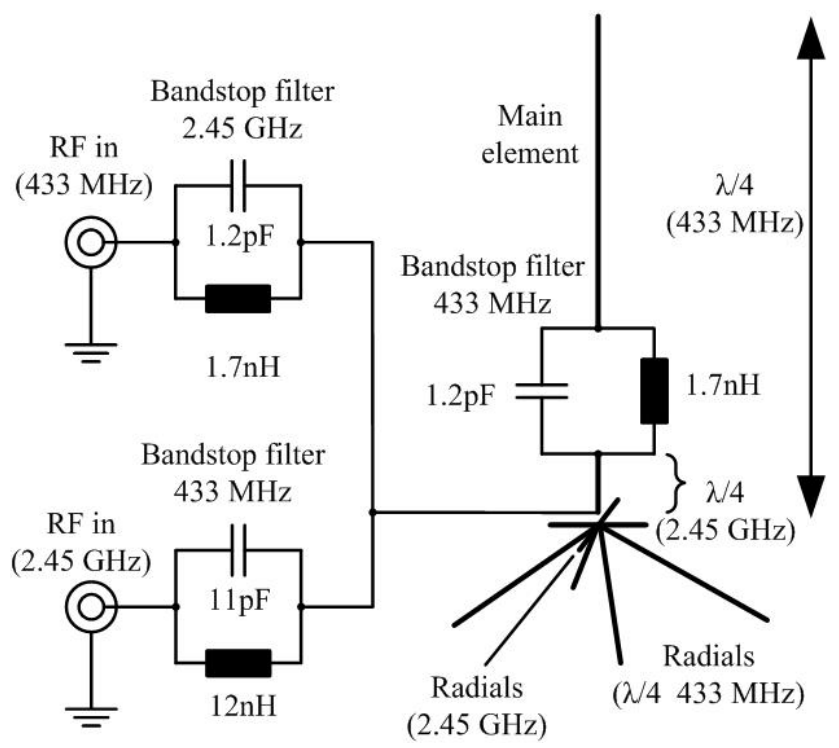

Fig. 4. Multi-resonant antenna for $2.45 \mathrm{GHz}$ and $433 \mathrm{MHz}$.

measurements the wave polarization is always vertical. This antenna is derived from the $\lambda / 4$ ground-plane antenna with 4 radials, each measuring $1 / 4$ wavelength. With the angle between the radials and the main element the impedance can be adjusted. The main element consists of a $\lambda / 4$ long silvered copper wire of $2 \mathrm{~mm}$ diameter (in terms of $433 \mathrm{MHz}$ ). On the lower end there is a band pass mounted that blocks the $2.45 \mathrm{GHz}$ signal. The position of this filter is about $\lambda / 4$ over the feeding point (in terms of $2.45 \mathrm{GHz}$ ). To allow separate matching for both frequencies to $50 \Omega$, short and long radials are attached. The long radials provide $50 \Omega$ at an angle of about $135^{\circ}$ for $433 \mathrm{MHz}$. With the short ones the impedance of the $2.45 \mathrm{GHz}$ antenna part is adjusted. The short radials have almost no influence to the impedance of the longer wavelength. With this separate radial configuration both impedances can be tuned to $50 \Omega$ separately.

The signals have to be combined without mutual impedance mismatch. This is achieved by band-pass filters in every signal path isolating one frequency against the other. After connecting the band pass to the antenna the impedance has slightly misaligned which has to be reversed by adjusting the radials again.

The phase centre is not exactly at the same point for both frequencies in vertical direction but it is much better than with two separate monopole antennas.

\section{Detection in case of single objects in the signal path}

To verify radiation effects, several measurements with simple objects were carried out in open space areas (Fig. 5). The transmitter (TX) emits continuous wave signals at both frequencies that travel to the receiver (RX) either directly or

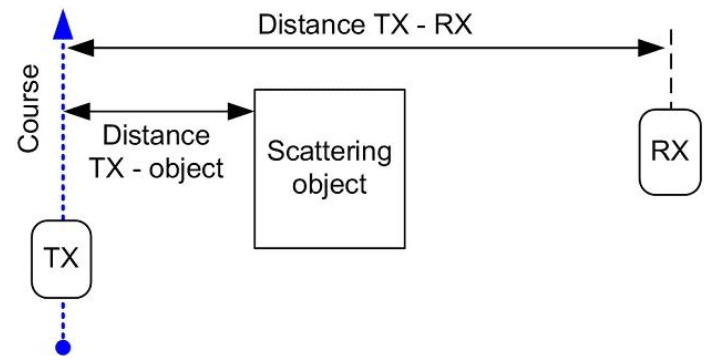

Fig. 5. Double edge diffraction at a car.
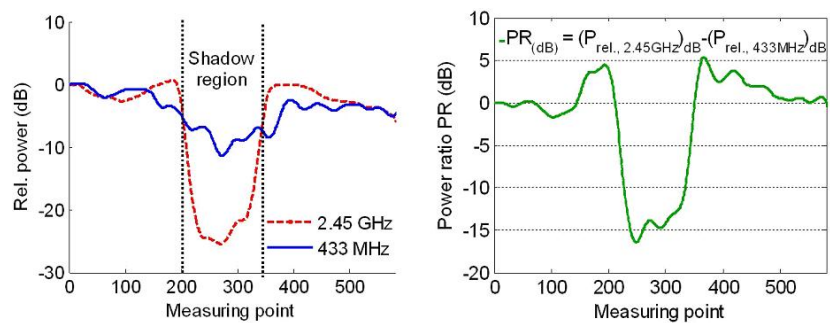

Fig. 6. Detection of a target hidden by a single object left: normalized received power at the two frequencies, below: power ratio for the detection (distance $\mathrm{TX}-\mathrm{RX}=36 \mathrm{~m}$, antenna-high $\mathrm{TX}=1.2 \mathrm{~m}$, antenna-high $_{\mathrm{RX}}=0.8 \mathrm{~m}$ ).

disturbed by an scattering object. A relative movement of the target, receiver or object causes a position-dependent signal power at the receiver antennas. This signal power is measured separately for both frequencies at RX. The scattering object used is a metal sculpture with sharp edges and dimensions of $2 \mathrm{~m}$ in each direction. Figure 6 shows the diffraction measurement with a distance of $35 \mathrm{~m}$ between the receiver and the target and $1 \mathrm{~m}$ distance between target and object. After measurement point (MP) 200, the target person gets invisible for the receiver, and there are only waves present at RX which are double diffracted at the edges of the scattering object. The $433 \mathrm{MHz}$ signal experiences only a small attenuation due to the object compared to the $2.45 \mathrm{GHz}$ one. The lower graph indicates the ratio in signal power of both frequencies. As the theory predicts, this value is about $15 \mathrm{~dB}$ if the target is hidden relating to $0 \mathrm{~dB}$ for line of sight (LOS). If the threshold is placed for instance at $-5 \mathrm{~dB}$, it is easy to figure out in which interval the object is hidden by the target.

At certain distances, strong peaks in signal power arise in the shadow region resulting from interference between waves diffracted around different sides of the scattering object which can be verified through comparing propagation paths. At scattering objects with big radii, creeping waves have been detected. This has been verified through calculation of ray propagation paths leading to points with destructive interference which agree with measured peaks in the diffracted wave signal (McNamara et al., 1989). 


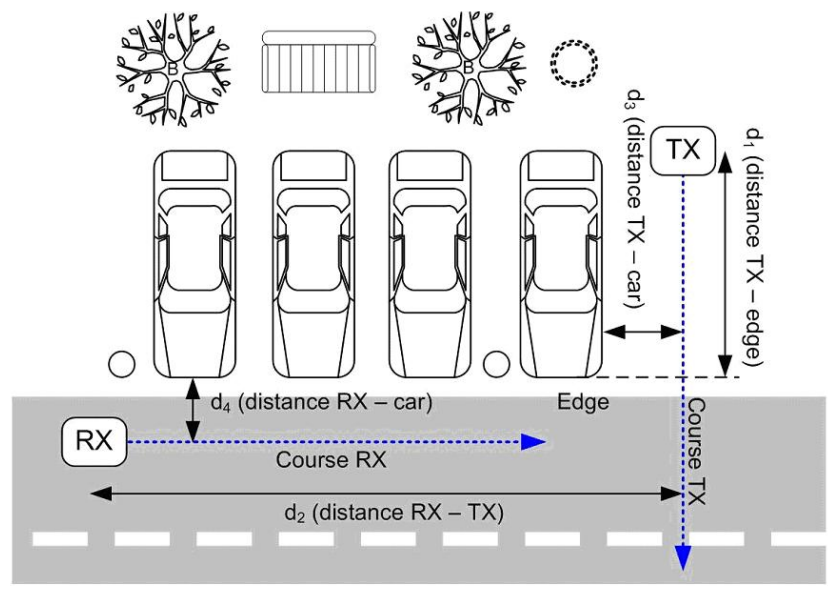

Fig. 7. Detection Measurement configuration for traffic situations.

\section{Detection of hidden targets in road traffic scenarios}

Several different road traffic scenarios have been analyzed to verify that the results are repeatable. Figure 7 shows a typical situation - parked cars at the roadside. In the first measurement, the emitter (TX) moves normal to the road in a certain distance to the parking car. The receiver (RX) is placed at a fixed position at the road. With this configuration, the scenario of a VRU moving from invisible positions to visibility is analyzed at a fixed position of the car. The measurement starts at $d 1=10 \mathrm{~m}$ corresponding to the measuring point (MP) zero in Fig. 8, where the target is hidden by the cars. After MP 390, the target person becomes visible for the receiver, and TX and RX are in LOS. By setting the threshold again at $-5 \mathrm{~dB}$, the target person can be identified as hidden (before MP 390) or as visible (afterwards). In this case, the detection has a reliability of about $95 \%$ for the target positions regarded. By averaging the signal over a certain time period, the error probability can be minimized.

Disturbing ripples of up to $20 \mathrm{~dB}$ arise due to constructive and destructive interference between direct and reflected waves in LOS case between diffracted and reflected waves or also between several diffracted waves in the shadow region. By averaging the signal power over a certain period of time, the power ratio between the two frequencies for hidden targets amounts always to about $15 \mathrm{~dB}$ relating to $0 \mathrm{~dB}$ for LOS. This result was confirmed in different measurements with moving targets and coincides with the theoretical calculation of Sect. 2.

The following measurement takes place in the same scenario indicated in Fig. 7 but with a moving receiver (RX) and an emitter at fixed positions. The receiver moves parallel to the street as can be seen from Fig. 7. During this measurement, the emitter remains at a fixed position. Two measurement results with different target - edge distances $\left(d_{1}\right)$ have been put together in Fig. 9: $d_{1, \mathrm{LOS}}=0 \mathrm{~m}$ (target just visible) and $d_{1, \text { HIDDEN }}=2 \mathrm{~m}$ (target hidden). With this configuration
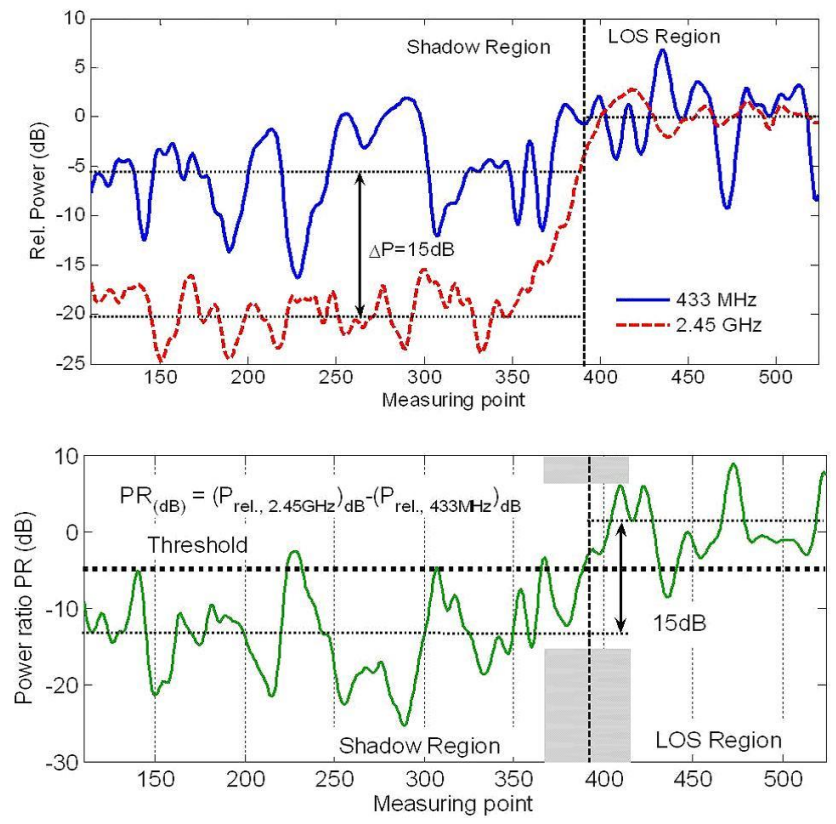

Fig. 8. Detection measurement of a moving target; above: normalized received power at the two frequencies, below: power ratio for the detection $\left(d_{2}=30 \mathrm{~m}, d_{3}=1 \mathrm{~m}, d_{4}=1 \mathrm{~m}\right.$, antenna-height $\mathrm{TX}=0.8 \mathrm{~m}$, antenna-height ${ }_{\mathrm{RX}}=0.6 \mathrm{~m}$ ).

the scenario of a moving car is analyzed in two cases - one with a hidden VRU and one with a VRU just visible. The movement starts at a target distance of $d_{2}=35 \mathrm{~m}$ (MP 0 ) and stops at $d_{2}=2 \mathrm{~m}$ (MP 400). At the last few meters there is a big misalignment of the antennas because TX is displaced from the RX moving path by $3 \mathrm{~m}$ (target hidden) or $1 \mathrm{~m}$ (target in LOS) respectively. So these values after MP 350 are not used for calculation of the mean value. In contrast to the prior measurements which show relative power levels this time the absolute signal power as a function of distance is shown in the upper graph of Fig. 9. The difference in the mean values of the power ratio in LOS and hidden case range with $18 \mathrm{~dB}$ in the same size as in the previous measurements. The signal difference is at all distances big enough for detection of hidden targets. By setting the threshold again to $-5 \mathrm{~dB}$, the detection has a reliability of more than $95 \%$.

The height of the antenna over the ground has a big impact on the measurement results. For instance if both antennas are at a height where LOS can be achieved through the windows of the cars, the signal difference is less because the signals experience double slit diffraction. For $2.45 \mathrm{GHz}$, the slit (car window) is big enough that at RX a signal power level is measured which is even higher as for LOS because of Fresnel diffraction. 


\section{Conclusion}

In this paper, the detection of hidden persons by using a transponder attached at the person is demonstrated. It has been shown that wavelength dependent diffraction effects can be utilised for the detection whether an emitter is hidden. In order to show that this effect is measurable without measurement equipment, a RF-measuring system was developed and built up for $2.45 \mathrm{GHz}$ and for $433 \mathrm{MHz}$. In open space areas with the used system configuration, detection of targets hidden by single big objects can be achieved up to $60 \mathrm{~m}$ distance between TX and RX. Here the power difference amounts to about $15 \mathrm{~dB}$ between hidden emitters relating to $0 \mathrm{~dB}$ for LOS confirming the theoretical calculation of Sect. 2.

Several target detection measurements in different road traffic scenarios have been carried out. The strong ripple in the power measurement arises due to multipath propagation. By averaging the signal over a certain period of time, the power ratio between the two frequencies for hidden targets amounts to about $12-18 \mathrm{~dB}$ relating to $0 \mathrm{~dB}$ for LOS. The detection whether a target is hidden behind a car is possible at any orientation of the car but depends from the height of the emitter. Relatively small objects like persons or street lamp poles in the signal path do not lead to detection which may be desirable. The results were confirmed in many different measurements with moving targets and also with moving receivers.

Multipath propagation causes strong interferences. Especially in situations with buildings or other objects at the roadside, a detection with-non averaged measurement values is in some cases impossible because of the strong ripple in signal power. A solution to overcome this problem is using diversity reception. In this case, for the reception of a continuous wave signal only, this can be accomplished by switching two or more antennas consecutively to one LNA and always taking the highest signal value.

An important aspect is to set the threshold at a level with the best detection reliability between LOS and hidden target. A variable threshold depending on other parameters such as distance between RX and TX which has to be measured simultaneously may be advantageous.

If the body of the target person turns into the signal path, both frequencies are attenuated up to $30 \mathrm{~dB}$. This attenuation does not arise at the same turning angle for both frequencies, so an additional power difference of more than $15 \mathrm{~dB}$ must be taken into account, too. For this reason, the error probability in detection for statistical target orientation increases significantly. Further investigations have to be made to determine the impact of the target person's orientation at different road traffic situations.
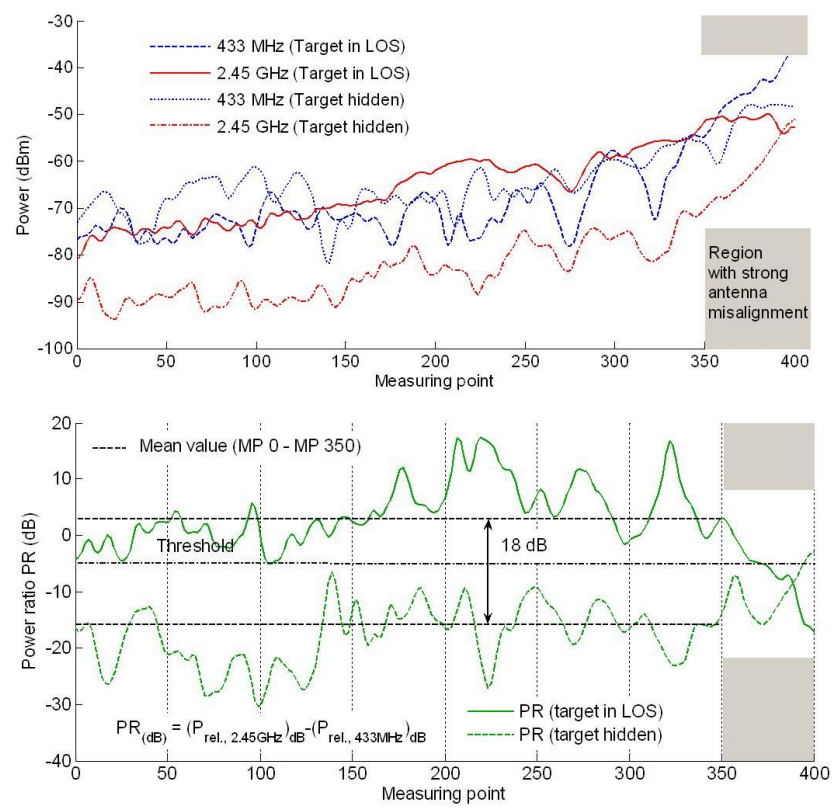

Fig. 9. Detection of a moving target, above: received power at the two frequencies, below: power ratio $\left(d_{3}=1 \mathrm{~m}\right.$ and $d_{4}=1 \mathrm{~m}$, $d_{1, \mathrm{LOS}}=0 \mathrm{~m}, d_{1, \mathrm{HIDDEN}}=2 \mathrm{~m}$ antenna-height $\mathrm{TX}_{\mathrm{TX}}=0.8 \mathrm{~m}$, antennaheight $_{\mathrm{RX}}=0.6 \mathrm{~m}$ ).

\section{References}

Borovikov, V. A. and Kinber, B. Y.: Geometrical theory of diffraction, Bath: Bookcraft, 1992.

Keller, J. B.: Geometrical Theory of Diffraction, J. Opt. Soc. Am., 5(2), 116-132, 1962.

McNamara, D. A., Pistorius, C. W. I., and Malherbe, J. A. G.: The Uniform Geometrical theory of diffraction, Boston: Artech House, 1989.

Rasshofer, R. H., Schwarz, D., Biebl, E., Morhart, C., Scherf, O., Zecha, S., Grünert, R., and Frühauf, H.: Pedestrian Protection System using Cooperative Sensor Technology, Proc. Advanced Microsystems for Automotive Applications AMMA 2007, 135145, Berlin, Germany, 2007. 